\title{
Efecto de la explotación forestal sobre la estructura, diversidad y composición florística de los palmitales de la Selva Atlántica en Misiones, Argentina
}

\author{
Sandra E. Chediack \\ Instituto de Ecología, Universidad Nacional Autónoma de México. Alcanfores 68 Fracc. Real del Monte, San \\ Cristóbal de las Casas, Chiapas, México, código postal 29299; chediack@miranda.ecologia.unam.mx
}

Recibido 27-III-2007. C Corregido 18-IX-2007. Aceptado 29-IV-2008.

\begin{abstract}
The effect of forest exploitation on structure, diversity, and floristic composition of palmitodominated Atlantic forests at Misiones, Argentina. The effect of forest exploitation - timber and palmito (Euterpe edulis, Palmae) extraction - on structure, diversity, and floristic composition of forests known as palmitals of the Atlantic Forest of Argentina was analyzed. These palmitals are located in Misiones (54 ${ }^{\circ} 13^{\prime}$ $\mathrm{W}$ and $\left.25^{\circ} 41^{\prime} \mathrm{S}\right)$. Three 1 ha permanent plots were established: two in the "intangible" zone of the Iguazu National Park (PNI), and another in an exploited forest site bordering the PNI. Three 0.2 ha non-permanent plots were also measured. One was located in the PNI reserve zone where illegal palmito extraction occurs. The other two were in logged forest. All trees and palmitos with $\mathrm{DBH}>10 \mathrm{~cm}$ were identified and $\mathrm{DBH}$ and height were measured. For each of the six sites, richness and diversity of tree species, floristic composition, number of endemic species, and density of harvestable tree species were estimated. The harvest of $E$. edulis increases density of other tree species, diminishing palmito density. Forest explotation (logging and palmito harvest) is accompanied by an increase in diversity and density of heliophilic species, which have greater timber value in the region. However, this explotation also diminishes the density of palmito, of endemic species which normally grow in low densities, and of species found on the IUCN Red List. Results suggest that forest structure may be managed for timber and palmito production. The "intangible" zone of the PNI has the greatest conservation value in the Atlantic Forest, since a greater number of endemisms and endangered species are found here. Rev. Biol. Trop. 56 (2): 721-738. Epub 2008 June 30.
\end{abstract}

Key words: Atlantic forest, Euterpe edulis, subtropical forest,forest management, protected areas, endemisms.

Los palmitales son bosques naturales que se encuentran en la Selva Atlántica y que se caracterizan por tener una alta densidad de palmito (Euterpe edulis Mart., Palmae) (Placci y Giorgis 1993). Esta palma unicaule se corta para extraer el "palmito" o "cogollo" comestible que se comercializa como alimento gourmet. Los palmitos, más los árboles de especies maderables, hacen del palmital uno de los bosques nativos de la Selva Atlántica interior de mayor valor económico.

La Selva Atlántica es una de las regiones del planeta con mayor biodiversidad y riesgo de desaparecer. Según Myers et al. (2000) esta selva es el cuarto "punto caliente de biodiversidad" en cuanto a necesidades de conservación por la excepcional concentración de especies endémicas que presenta y la alta tasa de desaparición de sus ambientes naturales. Se ha estimado que en ella existen alrededor de 20 000 especies de plantas vasculares de las cuales 8000 son endémicas (Myers 1988). Se estima que solo queda entre el 7 y el $8 \%$ de su superficie original (Galindo-Leal y Gusmao Câmara 2003). La superficie de selva remanente en Argentina es de aproximadamente $11300 \mathrm{~km}^{2}$, 
éste es el fragmento continuo más grande de Selva Atlántica y su estado de conservación es variable según los sitios (Holz y Placci 2003). Lo mismo sucede con los palmitales que están inmersos en ella. En términos de explotación, estos palmitales pueden clasificarse como: $i$ ) sin explotación, como los del área intangible del Parque Nacional Iguazú, ii) bajo aprovechamiento legal de palmitos y árboles maderables, como en algunas propiedades privadas, y iii) bajo extracción ilegal de palmitos, como en la zona de reserva del Parque y en propiedades privadas.

El aprovechamiento del palmito consiste en el corte de las palmeras con machete; el tronco y la corona de hojas quedan en el suelo y solo se retira el "palmito" que es el extremo apical de $1 \mathrm{~m}$ de largo aproximadamente. El "palmito" es llevado por una persona hasta el camino más cercano donde es colocado en camiones para su transporte hasta las envasadoras. Legalmente se permite el corte de palmitos con un DAP (diámetro del tronco a $1.3 \mathrm{~m}$ ) mayor a $10 \mathrm{~cm}$ (Ministerio de Ecología de la Provincia de Misiones, Argentina). El impacto del aprovechamiento de palmitos sobre la composición florística y estructura (densidad de árboles organizados según el tamaño de diámetro o altura del tronco) de la selva no ha sido estudiado.

El aprovechamiento forestal maderero en la zona de estudio comprende el corte de árboles con motosierras y el arrastre del tronco con cadenas tiradas por tractores. El corte de los árboles maderables puede provocar la muerte de otros árboles (Boot y Gullinson 1995), erosión del suelo y disminución en la biomasa de animales (Lammertink 2004, Wells et al. 2007). En los palmitales argentinos la ley permite el corte de determinadas especies de árboles con un DAP mayor a $50 \mathrm{~cm}$ (Ministerio de Ecología de la Provincia de Misiones, Argentina). El efecto del aprovechamiento maderero sobre la diversidad de especies arbóreas y la estructura del bosque ha sido más estudiado. Inmediatamente después del corte disminuye la densidad de árboles de todos los tamaños por la extracción de individuos reproductivos y por la mortalidad de renuevos producto de las labores de aprovechamiento (Boot y Gullison 1995, Cannon et al. 1998). El aprovechamiento forestal en los bosques tropicales puede producir una pérdida significativa en la riqueza de especies arbóreas (Cannon et al. 1998) o no (Panfil y Gullison 1998). Generalmente el aprovechamiento también puede alterar la composición de especies. Dependiendo del tamaño de las aberturas de dosel producto del aprovechamiento, habrá o no cambios en la composición de especies (ter steege et al. 1996, Sáenz et al. 1999). Si estas aberturas son relativamente pequeñas, favorecerán el crecimiento de plantas existentes sin producirse cambios significativos en el conjunto de especies presentes. Por el contrario, las aberturas grandes permitirán el reclutamiento, a partir de semillas, de especies heliófilas y una mayor mortalidad de especies tolerantes a la sombra. Es por esto que en los bosques explotados para la extracción maderera los componentes de la diversidad que más están amenazados son aquellas especies características de bosques en estadios avanzados de sucesión y bosques maduros (Burley y Guauld 1995). La riqueza, diversidad y composición específica arbórea después de un aprovechamiento también depende de la especie que está siendo extraída. Yodzis (1989) sugiere que el número de especies en una comunidad depende del grado de selectividad que tiene el factor mortalidad dentro de una comunidad y la dominancia de las especies seleccionadas. Siguiendo esta hipótesis, si el aprovechamiento no es selectivo y se cortan todas las especies o especies subdominantes se produciría un decremento en la riqueza de especies. $\mathrm{Si}$, en cambio, la mortalidad afecta a especies dominantes, el resultado será un aumento en la riqueza de especies. Para una comunidad dominada por una especie, cualquier reducción en la dominancia permite la coexistencia de más especies. Por lo tanto al desaparecer la especie dominante, la comunidad podría estructurarse ya sea de manera más equitativa (aumentando su diversidad), o de forma heterogénea si otra especie se convierte en dominante (disminuyendo su diversidad). 
En este trabajo se analiza el efecto del aprovechamiento forestal (de palmito y de especies maderables) sobre la estructura, diversidad y composición florística arbórea de los palmitales de la Selva Atlántica de Argentina.

\section{MATERIALES Y MÉTODOS}

Área de estudio: Se trabajó en palmitales ubicados alrededor de las coordenadas $54^{\circ} 13^{\prime}$ $\mathrm{O}$ y $25^{\circ} 41^{\prime} \mathrm{S}$, en zona norte de la provincia de Misiones, Argentina. El clima es subtropical húmedo sin estación seca marcada. La temperatura media anual es $20.3^{\circ} \mathrm{C}$, la temperatura media del mes más cálido (febrero) es $25.7^{\circ} \mathrm{C}$ y la del más frío (julio) $8.4^{\circ} \mathrm{C}$, registrándose días con temperaturas por debajo de $\operatorname{los} 0^{\circ} \mathrm{C}$. La precipitación media anual es de $\sim 1,600 \mathrm{~mm}$ (Crespo 1982). Durante la época estival son comunes las tormentas eléctricas de carácter torrencial. También son frecuentes los tornados con fuertes vientos que destruyen la vegetación y cuyas cicatrices pueden observarse en imágenes satelitales 15 años después de ocurridos (Dyer 1988). Según Gatti (com pers.) los palmitales misioneros se desarrollan en las zonas con menor incidencia de heladas invernales, entre los 200 y $500 \mathrm{msnm}$ y en las orillas de grandes ríos.

Los palmitales muestreados son:

- El Parque Nacional Iguazú (PNI): Este parque se decretó en 1934 y el uso anterior se restringió al corte de árboles de 4 especies, Cedro (Cederela fissilis, Melicaceae), Peteribí (Cordia trichotoma, Boraginaceae), Lapacho (Tabebuia heptaphylla, Bignoniaceae) e Incienso (Myrocarpus frondosus, Leguminosae), y solo de individuos mayores a $55 \mathrm{~cm}$ de diámetro del tronco (Devoto y Rothkugel 1936). En el Parque se trabajó en dos áreas, una de ellas en la zona intangible, donde solo se realizan actividades de vigilancia y científicas. En este trabajo se ha usado la estructura y diversidad de los palmitales de ésta zona como referencia para determinar los cambios que produce el aprovechamiento del bosque. Su importancia en conservación y los servicios ambientales que brinda se detallan en Chediack (2006). En esta zona intangible se trabajó en dos sitios elegidos al azar denominados parcela permanente $1(P P 1)$ y parcela permanente 2 (PP2).

La otra área de estudio dentro del Parque se ubicó en la zona de reserva, en el Palmital denominado El Cruce. En este palmital, a pesar de los esfuerzos de protección del PNI, se producen extracciones ilegales de palmito frecuentemente. Sin embargo, debido a la presión de la acción ilegal, los furtivos solo cortan individuos con los palmitos de muy buena calidad. Un muestreo del área afectada por un corte ilegal de palmitos dentro del Parque indicó que la mayor cantidad de palmitos cortados ilegalmente tenían entre los 5 y $10 \mathrm{~m}$ de altura (Placci et al. 1992).

- El establecimiento forestal San Jorge (ESJ): Propiedad privada que limita al sur del PNI, fue aprovechado forestalmente en 1987 y no se tiene conocimiento de la historia de uso anterior a dicho año (Gotz y Dalprá, com. pers.)

- El establecimiento forestal del Ejército Argentino (EJEA): El sitio donde se trabajó sufre explotación ilegal de palmitos constantemente. En 1987 se realizó aprovechamiento de palmito y un enriquecimiento del bosque con semillas de palmito, producto de esto es una alta densidad de renuevos de esta especie en el sotobosque (Fuget y Montalvo, com. pers.).

- El Yaguareté (Y): Propiedad privada que forma parte del límite Este del PNI. Se realiza aprovechamiento forestal legal, desde 1995 hasta el momento de muestreo se extrajeron 100 árboles maderables y 10 225 palmitos. La propiedad ha sido declarada Refugio de Vida Silvestre en el 2002 
por la Fundación Vida Silvestre Argentina (Schiaffino et al. 2001).

Muestreo: El trabajo de campo se realizó en enero, febrero y marzo de 1997 y julio de 1998, es decir 4 meses en total. La composición florística arbórea fue muestreada en 3 ha de parcelas permanentes y 0.6 ha de parcelas no permanentes. Para el marcado y medición de las parcelas se siguió el método utilizado por el Instituto Smithsoniano y el Programa de reservas del hombre y la biosfera (Dallmeier 1995). En cada parcela permanente, todas las plantas (árboles, palmeras y lianas) con diámetro de tronco a una altura de $1.3 \mathrm{~m}$ del suelo (DAP) igual o mayor a $10 \mathrm{~cm}$ fueron marcadas con chapas metálicas numeradas y se registró la especie, altura total y perímetro de tronco. El muestro no permanente consistió en 5 parcelas de $10 \times 40 \mathrm{~m}$ totalizando 0.2 ha en cada sitio. En éstas no se marcaron los individuos. En el caso de no reconocerse la especie, se recolectaron ejemplares de herbario que fueron depositados en el herbario del CIES (Centro de Investigaciones Ecológicas Subtropicales del PNI) y enviadas al Instituto de Botánica del Noreste para su identificación. Se ubicaron dos parcelas permanentes en el área intangible del PNI (PN1 y PN2) y una en el ESJ; estas parcelas medían una ha cada una, y son parte de un estudio de dinámica forestal a largo plazo de palmitales. Los muestreos no permanentes se realizaron en El Cruce, EJEA y en Y.

Análisis de los datos: Para cada sitio se estimó la diversidad y riqueza específica utilizando el programa Spade (Chao y Shen 2003b). Este programa utiliza un método no- paramétrico para estimar el índice de Shannon cuando se espera que existan especies no registradas en un muestreo (Chao y Shen 2003a). Los índices de Shannon de cada sitio se compararon con la prueba t (Zar 1996) y la corrección de Bonferroni (Sokal y Rohlf 1995).

Para cada sitio se estimó densidad, área basal, altura promedio y máxima y estructura diamétrica y altimétrica del bosque considerando a las plantas con DAP $>10 \mathrm{~cm}$ (Dallmeier 1995).
Para comparar las estructuras diamétricas de los palmitales se clasificó a los individuos (palmitos, árboles y lianas) en las siguientes categorías diamétricas:1) $10 \leq \mathrm{DAP}<20 \mathrm{~cm} ; 2$ ) $20 \leq \mathrm{DAP}<50 \mathrm{~cm}$; 3) $50 \leq \mathrm{DAP}<100 \mathrm{~cm}$; 4) $\mathrm{DAP} \geq 100 \mathrm{~cm}$. Para cada uno de los 6 sitios de estudio y para cada categoría diamétrica se estimó el número promedio de individuos en 0.2 ha. Para determinar si existían diferencias significativas en la densidad entre sitios considerando cada categoría se las comparó con la prueba de Duncan. Se calculó la densidad promedio de las especies maderables por categoría de tamaño por sitio. En esta comparación no se incluyó la categoría 4 porque los árboles de ese tamaño no son maderables. Se utilizó la prueba de Duncan para determinar si existían diferencias significativas en la densidad de árboles maderables entre sitios. La lista de especies aprovechadas se obtuvo del Ministerio de Ecología de la Provincia de Misiones, Argentina (www.misiones.gov.ar).

Para determinar la relación entre la densidad promedio de palmito y la de otras especies se realizó una correlación de Spearman. Para ésta solo se utilizaron los datos registrados dentro del Parque Nacional donde no hay aprovechamiento de especies arbóreas.

Para determinar como varía la diversidad específica con el aprovechamiento se realizó una correlación de Spearman entre la densidad de palmitos promedio en 0.2 ha con el índice de Shannon de cada sitio. Se consideró la densidad de palmito como una medida de aprovechamiento del bosque, cuanto menor es su densidad se considera al bosque más intervenido. También se correlacionó la riqueza de especies en cada una de las hectáreas de las parcelas permanentes y la estimada para los sitios no permanentes con la densidad de palmitos.

Las especies registradas se organizaron según el criterio de conservación de la UICN (Unión Internacional para la Conservación de la Naturaleza) que les corresponde en la "Lista roja de especies amenazadas". También se catalogaron como endémicas o no endémicas de la Selva Atlántica. Para esto se consultó el catálogo de las plantas vasculares de la Argentina 
(Zuloaga y Morrone 1999), la base de datos del jardín botánico de Missouri VAST (http:// mobot.mobot.org/W3T/search/vast.html) y la base BIOCORES (Conservación de la biodiversidad, restauración y uso sustentable en paisajes fragmentados) del Programa de las naciones unidas para el medio ambiente y el Centro de monitoreo de la conservación.

\section{RESULTADOS}

La estructura y diversidad de los palmitales estudiados se resumen en los cuadros 1 y 2 .

La mayor densidad de individuos (palmitos, árboles y lianas) se registró en el Parque Nacional. Ello se debe al alto número de palmitos, los cuales comprenden el $62 \%$ de los individuos con DAP $>10 \mathrm{~cm}$ en el área intangible del parque. La alta densidad de palmas comprende el $64.4 \%$ de los individuos si se considera las dos especies presentes: Arecastrum romanzoffianum y E. edulis. Este porcentaje es mayor al registrado en otros bosques neotropicales. Por ejemplo Gentry y Terborgh (1990) describen bosques donde las palmas imparten una fisonomía distintiva al bosque al constituir entre 1/6 y 1/4 de los árboles con troncos mayores a $10 \mathrm{~cm}$ de diámetro. El estrato formado por los palmitos en el PNI le da una fisonomía particular al bosque con un dosel continuo formado por las copas de esta palma.

La densidad de árboles y lianas (es decir si al número total de individuos se le resta el número de individuos de palmito) es mayor en los sitios aprovechados. En el EJEA no se registraron palmitos con $\mathrm{DAP}>10 \mathrm{~cm}$, aunque si de menor tamaño que no han sido considerados en este trabajo. En todos los sitios el DAP de los palmitos fue menor a $19 \mathrm{~cm}$.

La mayor área basal se encuentra en la PP1 del PNI que es casi 1.5 veces mayor a la de los otros sitios. Esto se debe a la alta densidad de individuos y a la presencia de árboles emergentes con DAP $>100 \mathrm{~cm}$ los cuales solo se observaron en el Parque Nacional. Estos árboles emergentes del dosel pertenecen a Aspidosperma polyneuron (Apocynaceae) conocida localmente como Palo Rosa.

La prueba de Duncan arrojó diferencias significativas en el número promedio de individuos

Estructura de la selva de cada una de las tres hectáreas de parcelas permanentes. Para la estimación de la altura, riqueza y diversidad de especies no se consideró a las lianas

Forest structure in each of the 3 ha of permanent plots. Lianas were not taken into account to estimate the height, richness and diversity of species

Parámetros estructurales

Densidad (Número de individuos ha ${ }^{-1}$ )

Densidad de palmitos (Número de individuos ha-1)

Densidad de árboles y lianas (Número individuos ha-1)

Área basal $\left(\mathrm{m}^{2} \mathrm{ha}^{-1}\right)$

Altura promedio (m)

Altura máxima (m)

Riqueza (Número de especies ha ${ }^{-1}$ )

Diversidad (Índice de Shannon)
Sitios

$\begin{array}{ccc}\text { PP1 } & \text { PP2 } & \text { ESJ } \\ 853 & 730 & 447 \\ 570 & 420 & 49 \\ 283 & 310 & 398 \\ 41.66 & 28.98 & 28.74 \\ 13 & 12 & 13 \\ 45 & 41.5 & 30 \\ 55 & 58 & 70 \\ 1.86 & 2.21 & 3.7\end{array}$




\section{CUADRO 2}

Estructura de los bosques en los muestreos no permanentes. No se consideró a las lianas para la estimación de las alturas y la riqueza de especies

TABLE 2

Forest structure in non permanent samples. Lianas were not taken into account to estimate the height and richness of species

Parámetros estructurales

Densidad (Número de individuos $0.2 \mathrm{ha}^{-1}$ )

Densidad de palmitos (Número de individuos $0.2 \mathrm{ha}^{-1}$ )

Densidad de árboles y lianas (Número de individuos $0.2 \mathrm{ha}^{-1}$ )

Área basal $\left(\mathrm{m}^{2} 0.2 \mathrm{ha}^{-1}\right)$

Altura promedio (m)

Altura máxima (m)

Riqueza (Número de especies $0.2 \mathrm{ha}^{-1}$ )

Diversidad (Índice de Shannon)
Sitios

$\begin{array}{ccc}\text { Cruce } & \text { Y } & \text { EJEA } \\ 142 & 116 & 116 \\ 62 & 40 & 0 \\ 80 & 76 & 116 \\ 5.34 & 4.51 & 5.86 \\ 11 & 11 & 12 \\ 20 & 25 & 21 \\ 34 & 33 & 30 \\ 2.72 & 3.94 & 3.13\end{array}$

entre los sitios para todas las categorías diamétricas excepto la categoría 3 (Fig. 1).

La densidad promedio de individuos en los tres sitios del Parque Nacional y el Y es similar, a excepción de que existe una mayor densidad de emergentes en la PP1 que en el Cruce y Y.
La estructura del bosque del ESJ se diferencia de la del Parque por la baja densidad de individuos en la categoría 1, principalmente de palmitos. El EJEA posee una estructura diferente a los otros sitios ya que carece de emergentes y no tiene palmitos.

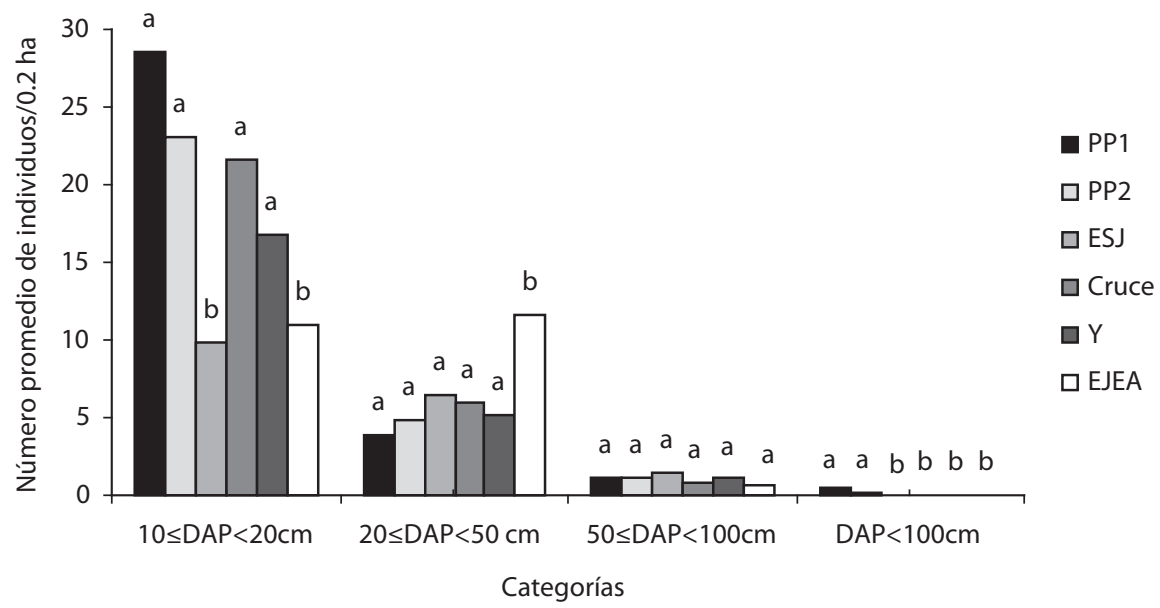

Fig. 1. Número promedio de individuos en cada categoría diamétrica en los 6 sitios estudiados. Dentro de cada categoría, los sitios con la misma letra no difieren significativamente entre sí al 0.05 (Prueba de Duncan).

Fig. 1. Average number of individuals in each diameter category in the six sites studied. Within each category, sites with the same letter do not differ significantly $(\mathrm{p}<0.05)$ among each other (Duncan Test). 
La densidad de árboles de especies maderables con categorías de tamaño 1 y 2 es mayor en el ESJ y en EJEA (Fig. 2).

En los sitios del PNI se observa un estrato de copas de palmitos entre los 5 y los $18 \mathrm{~m}$, teniendo la mayoría de los palmitos alrededor de $12 \mathrm{~m}$ de altura. En los sitios aprovechados forestalmente este estrato no es conspicuo. En todos los sitios los árboles con alturas mayores a $\operatorname{los} 18 \mathrm{~m}$ son escasos y en los sitios aprovechados desaparecen, por lo que E. edulis forma parte del dosel y no sólo del sotobosque (Figs. $3)$. Es posible que los sitios aprovechados posean una mayor luminosidad debido a la desaparición de la cobertura continua que producen las copas de los palmitos, sin embargo esto no ha sido medido en el marco de este trabajo.

Dentro del Parque Nacional donde hay menor densidad de palmitos tanto naturalmente (PP2) como por aprovechamiento ilegal (Cruce), se registra una mayor densidad de árboles y lianas (Spearman $=-1, p<0.001$, Fig. 4).

La diversidad expresada con el índice de Shannon muestra una correlación inversa con el número de palmitos (Spearman=-0.94, $\mathrm{p}=0.004$, Fig. 5), pero la diversidad en EJEA es menor a la esperada según esta relación. La riqueza de especies no muestra ningún patrón (Spearman $=0.14, \mathrm{p}=0.787$ ).

Se encontraron diferencias significativas $(\mathrm{p}=0.003)$ entre los siguientes pares de índices de Shannon: PP1- ESJ $(\mathrm{t}=5.07)$, PP1EJEA ( $\mathrm{t}=3.41)$, PP2- ESJ ( $\mathrm{t}=3.39)$, PP2- EJEA $(\mathrm{t}=2.05)$, Cruce - ESJ $(\mathrm{t}=2.38)$ y ESJ - EJEA $(t=3.49)$. Es decir, la diversidad es significativamente mayor en el ESJ que en el Parque y en EJEA, y en este último sitio es mayor que en el Parque pero menor que en ESJ.

El número total de familias de especies arbóreas registradas considerando todos los sitios fueron $37 \mathrm{y}$ el de especies fue 96. Cuatro especies pudieron ser identificadas solo hasta el nivel de género un Solanum, otra del género Cestrum, y dos del género Ocotea. Asimismo el género Ficus posiblemente incluya a varias especies. Las lianas incluyen a varias familias y especies que no fueron identificadas para este trabajo. La lista de especies, la familia a la que pertenecen, su densidad, si son endémicas, si son aprovechables forestalmente y si están en la lista roja de la UICN se resume en el Anexo 1.

De las especies registradas 28 son endémicas de la Selva Atlántica. En el Parque Nacional Iguazú se registraron 71 especies, de éstas 21

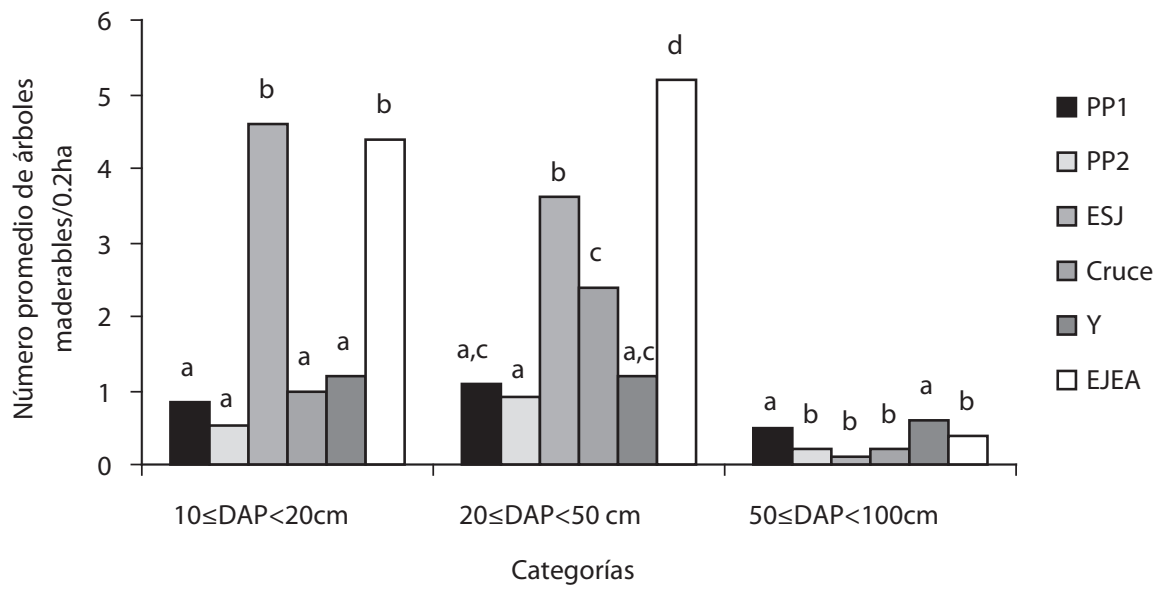

Fig. 2. Número promedio de árboles de especies maderables en cada categoría diamétrica en los 6 sitios. Dentro de cada categoría, los sitios con la misma letra no difieren significativamente entre sí al 0.05 (Prueba de Duncan).

Fig. 2. Average number of timber tree species of each diameter category in the six sites. Within each category, sites with the same letter do not differ significantly $(\mathrm{p}<0.05)$ among each other (Duncan Test). 

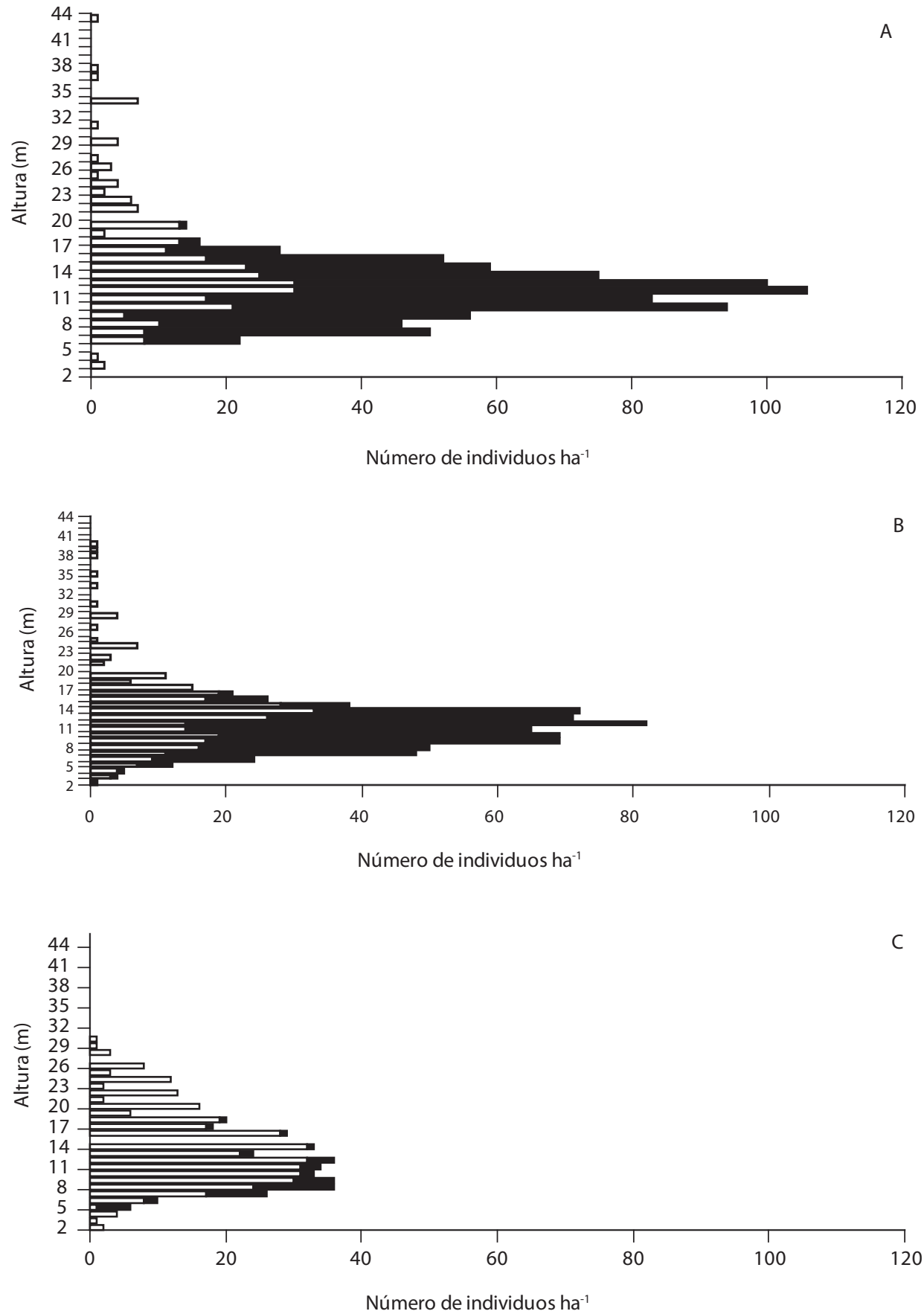

Figs. 3. Número de individuos clasificados en categorías altimétricas. En blanco los árboles y lianas. En negro los palmitos. Observar las diferencias en las escalas de las ordenadas y las abscisas de las graficas. A) PP1. B) PP2. C) ESJ. D) Cruce. E) Y. F) EJEA. 


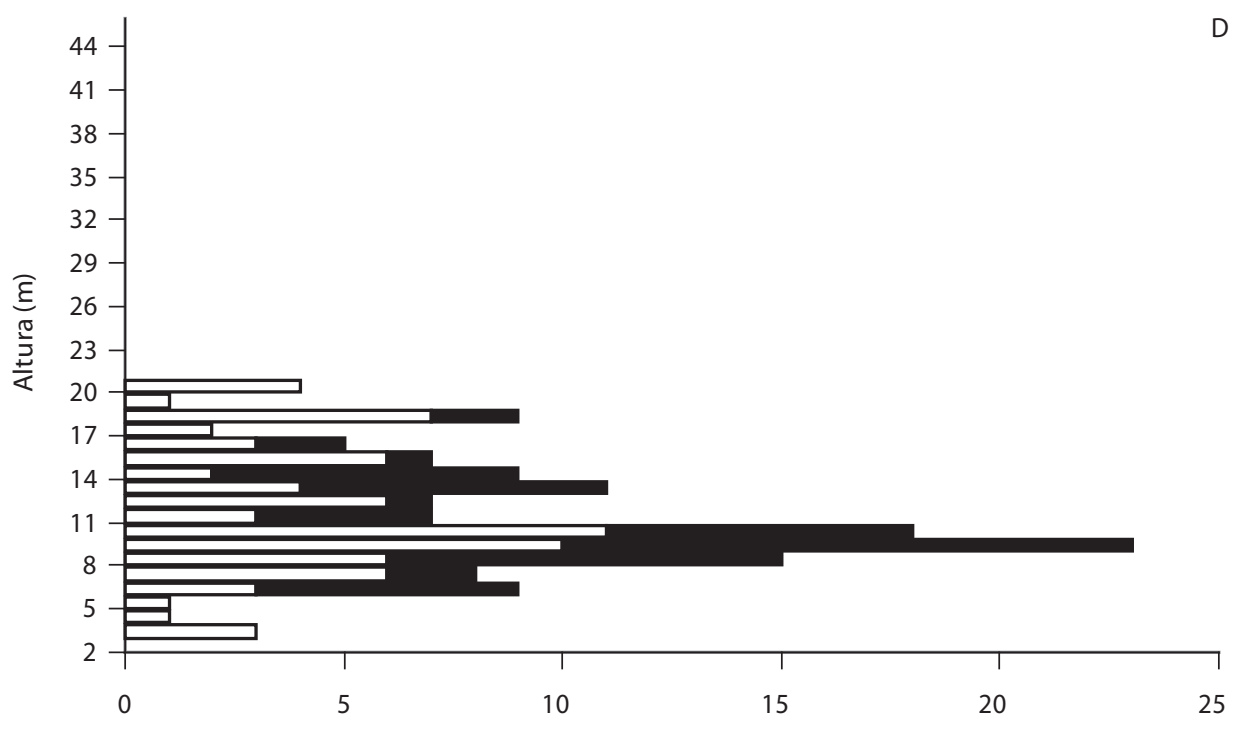

Número de individuos en 0.2 ha

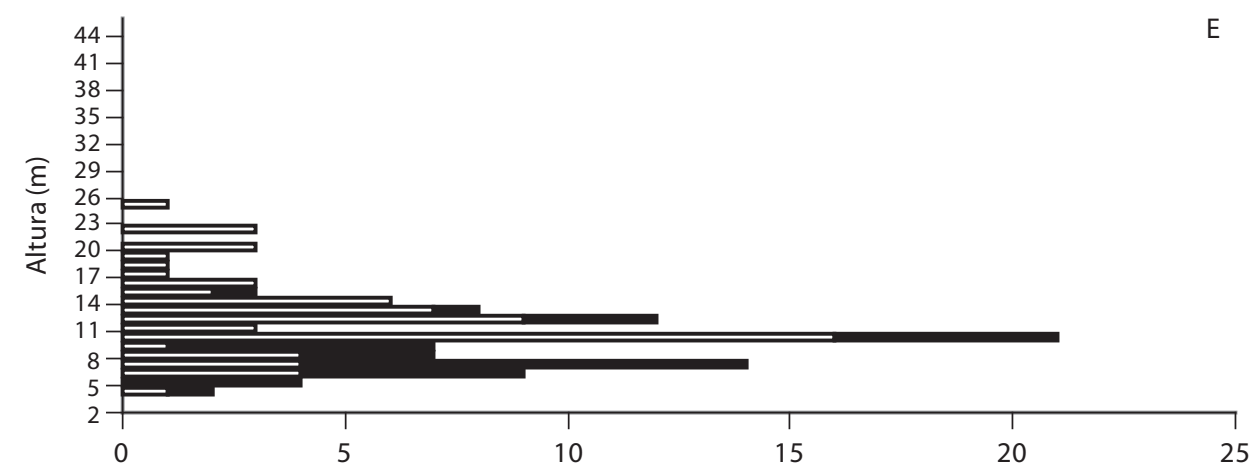

Número de individuos en 0.2 ha

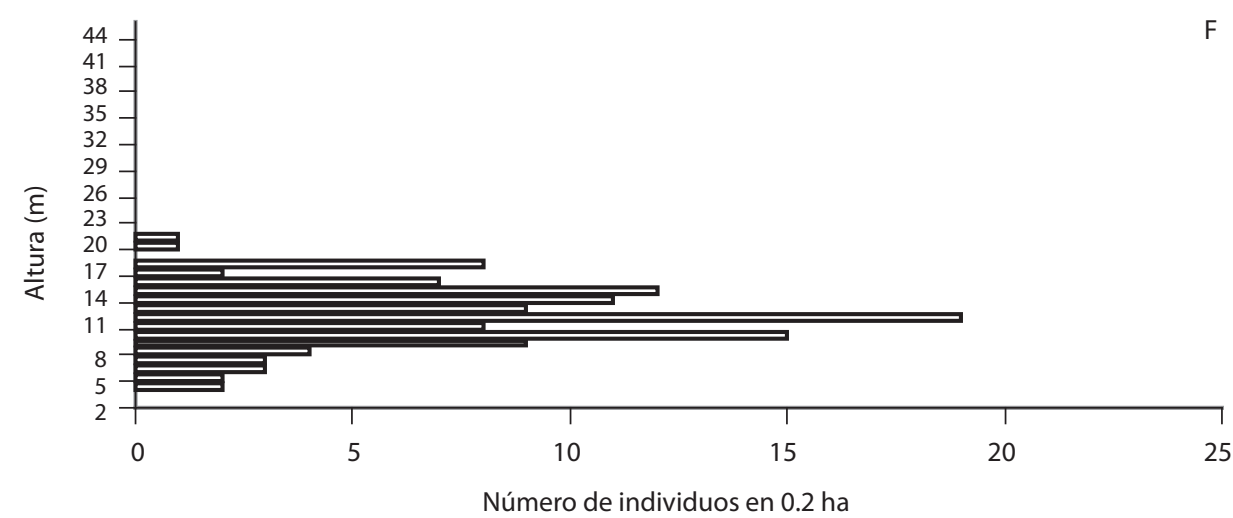

Figs. 3. Number of individuals classified in alimetric categories. Trees and lianas in white. palmitos in black. Scales in horizontal and vertical axes differ between graphs. A) PP1. B) PP2. C) ESJ. D) Cruce. E) Y. F) EJEA. 


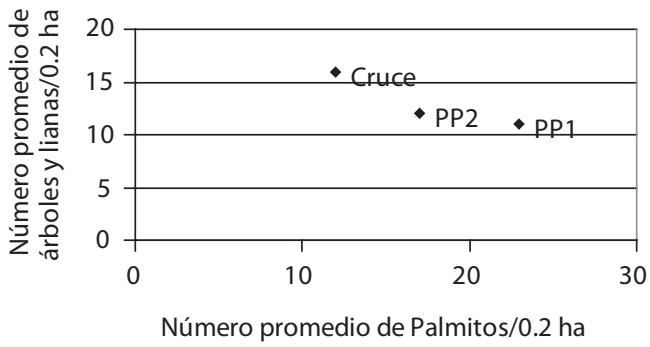

Fig. 4. Relación negativa entre la densidad promedio de palmitos/0.2 ha y de árboles y lianas en sitios sin aprovechamiento forestal maderable.

Fig. 4. Negative relation between the average number of palmito individuals and that of other species in 0.2 ha in sites without timber extraction.

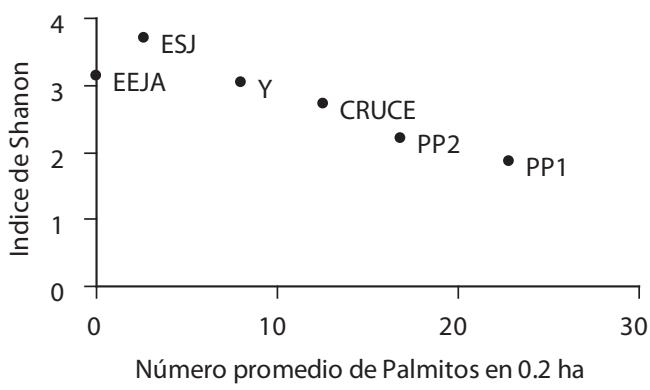

Fig. 5. Correlación negativa entre el índice de Shannon y la densidad promedio de palmitos.

Fig. 5. Negative correlation between the Shannon Index and average density of palmitos.

no se observaron en los sitios aprovechados. Dos de las 21 no pudieron ser identificadas por lo que no podemos determinar su distribución. De las 19 restantes 8 son endémicas de la Selva Atlántica. De las 16 especies registradas solamente en el palmital del área intangible del Parque, 14 están representadas por solamente uno ó dos individuos. De éstas, cinco son endémicas de Selva Atlántica: Banara parviflora (Flacourtiaceae), Cestrum euanthes (Solanaceae), Styrax leprosus (Styracaceae), Albizia edwallii (Leguminosae) y Pilocarpus pennatifolius (Rutaceae). En los sitios fuera del Parque se registraron 76 especies 27 de éstas no las encontramos en los muestreos del Parque. De las 27 seis son endémicas de Selva Atlántica.

Del total de las especies muestreadas 6 se encuentran en el libro rojo de la UICN. Una de ellas es $A$. edwallii la cual está considerada como vulnerable (World Conservation Monitoring Centre 1998 en UICN 2004) y estuvo representada por dos individuos en el Palmital intangible del Parque. El Incienso (Myrocarpus frondosus) está catalogado como $\mathrm{DD}$, es decir, no hay datos suficientes para poder ser clasificada inequívocamente en el libro rojo (Prado1998 en UICN 2004). Esta especie se presentó en las parcelas permanentes del Parque y en el ESJ pero no en los otros sitios aprovechados lo que quizás sugiere extracción debido a su naturaleza maderable. Tres especies están clasificadas como en peligro. Según la UICN estar en peligro significa que si no se revierte su situación actual, enfrentan un alto riesgo de extinción en los próximos 10 años. Estas son $A$. polyneuron, Balfourodendron riedelianum y Cedrela fissilis (Americas Regional Workshop 1998 en UICN 2004). Las tres especies fueron encontradas en sitios con y $\sin$ aprovechamiento forestal y en relativamente altas densidades. Solanum granulosum-leprosum está clasificado como en bajo riesgo en la subcategoría de dependiente de conservación de programas específicos (Carvalho 1998 en IUCN 2004). Esta especie ha sido registrada solo en las parcelas del área intangible del Parque.

\section{DISCUSIÓN}

El aprovechamiento forestal produce cambios en la estructura y diversidad de los bosques no solo por el corte y muerte de los árboles y palmas y la consecuente variación en luminosidad del bosque. Sino también porque el aprovechamiento incluye abertura de caminos, cacería de subsistencia de los obreros, otros cambios de factores ambientales y hasta de factores aleatorios difícilmente estimables. Los palmitales de la Selva Atlántica Argentina 
presentan estructuras, diversidad y composición florística diferentes dependientes de su manejo.

El manejo de protección que se realiza en la zona intangible del Parque asegura la protección de las especies sensibles al aprovechamiento forestal. En él se registraron especies que naturalmente se presentan en bajas densidades, solo en sitios sin aprovechamiento y que son endémicas de la Selva Atlántica. Estas tres características deberían tomarse en cuenta al catalogar las especies según su estado de conservación. Exceptuando a Albizia edwallii las especies registradas que tienen estas 3 características no se encuentran listadas en la lista roja de la UICN y quizá deberían incluirse (ver Anexo 1).

En el Cruce, donde solo se ha extraído palmito, la estructura del bosque es similar a la registrada en la zona intangible, sin embargo hay una mayor densidad de árboles. La menor densidad de palmito, la especie dominante, permite una mayor abundancia de otras especies cumpliéndose la hipótesis de Yodzis (1989). El mayor número de árboles y lianas cuando hay menor densidad de palmitos podría tener origen en que existen especies más exitosas que el palmito colonizando las pequeñas aberturas producto del corte o caída de individuos de esta palma. También puede influir la falta o disminución de individuos reproductivos de palmito que son los que estarían asegurando la regeneración de la especie. Por último es posible que entre las causas de esta relación intervengan otros factores como la relación entre la abundancia de semillas de palmito y la de semillas de otras especies arbóreas en el momento en que se realiza el aprovechamiento forestal. Begon et al. (1988) sugieren que en ambientes estacionales las especies que colonizan un espacio vacío varían con la estación. El estudio del efecto en la estructura y composición del bosque según la época de aprovechamiento es un proyecto que podría dar información para el manejo de los bosques misioneros.

El aprovechamiento forestal maderero y de palmito produce estructuras y composiciones florísticas diversas según cómo se realice. En Y los aprovechamientos fueron realizados, o en su defecto, controlados por los propietarios, quienes intentan aplicar técnicas de bajo impacto similares a las propuestas por Sist (2000). Estas técnicas se basan en planeación de los aprovechamientos minimizando las aberturas del dosel. La estructura diamétrica en general y la compuesta por las especies maderables fueron similares a las del Parque, pero la densidad de especies maderables es menor a la registrada en ESJ o en EJEA.

En los sitios donde no se aplican técnicas de aprovechamiento de bajo impacto (ESJ y EJEA), la estructura diamétrica, la composición florística y altimétrica y la diversidad son diferentes a lo registrado en PNI o Y. En ellos la baja densidad de palmito se traduce en un bosque de tipo mixto con una mayor equiparidad. Hay una mayor densidad de otras especies arbóreas especialmente las especies de valor forestal como Cedrela fissilis, Nectandra sp., Balfourodendron riedelianum y Cabralea canjerana. Aunque sus individuos presentaban aun un DAP menor al límite de corte legal de extracción, en el futuro el valor económico del bosque se incrementará. En EJEA se registró una menor diversidad que en ESJ tal vez producto de la alta frecuencia e intensidad de extracción de recursos que estaría empobreciendo el bosque. Whitmore (1998) sugirió que en determinados casos, dentro de los bosques tropicales puede suceder que ante la entrada repentina de luz, las especies que pueden responder rápidamente como Cecropia sp. cubren las aberturas y no permiten el establecimiento de otras plantas. Placci y Giorgis (1993) sugirieron que en algunos bosques del PNI podría estar perdiéndose la estructura forestal por que la caña nativa Chusquea ramossisima invade los claros y tendría un efecto inhibidor sobre la regeneración de otras especies. A medida que se mueren los árboles grandes, los claros son colonizados por esta caña y el bosque podría transformarse paulatinamente en una formación uni-específica de Ch. ramossisima. Dado que el aprovechamiento forestal produce aberturas del dosel y elimina el estrato formado por la copa de los palmitos, se espera que la 
luminosidad del bosque sea mayor, y, por lo tanto, también se espera que sea más suceptible al proceso sugerido por Placci y Giorgis. El estudio del origen de este tipo de formaciones vegetales, su dinámica temporal y los métodos de control constituyen un proyecto prioritario para el manejo de la Selva Misionera ya que estas formaciones disminuyen los valores económico y de conservación de la selva.

En conclusión el tipo de manejo que se realice en el bosque dependerá de cual sea el destino que se quiera para el bosque. En caso de que sea un bosque destinado a ser aprovechado dependerá de los objetivos de su propietario y el tipo de productos que este quiera obtener. Es decir un productor podría decidir si un bosque continuará siendo un palmital con E. edulis como especie dominante y que produzca principalmente palmito, o un bosque de tipo mixto con aprovechamiento menor de palmito pero mayor de especies maderables. $\mathrm{La}$ decisión del propietario seguramente no solo depende de las condiciones ambientales y del bosque que posee sino también de otros factores que el tendrá que evaluar como los turnos de corte de las especies, la rentabilidad de los productos obtenidos, la distancia al aserradero o la envasadora, la evaluación del riesgo que sus palmitos sean extraídos ilegalmente, la maquinaria con la que cuenta. etc.

Los bosques sin aprovechamiento solo se encuentran dentro del Parque Nacional Iguazú en sitios alejados de los caminos y los ríos por donde entran furtivos. Es en ellos donde aún se encuentra una buena representación de la Selva Atlántica con la presencia de muchas de las especies endémicas y amenazadas. Es necesario asegurar este tipo de ambiente mejorando el sistema de protección y favoreciendo estudios que permitan obtener información de referencia para comparar con sitios aprovechados.

El aprovechamiento forestal produce cambios en la estructura, diversidad y composición florística de los palmitales. Sin embargo, al contrario de la creencia popular y de muchos ecólogos, un buen manejo forestal en vez de empobrecer su riqueza maderera puede acrecentarla.

\section{AGRADECIMIENTOS}

Este trabajo no habría podido realizarse sin la colaboración de J. Herrera, M. G. Gatti, L. Malmierca, K. Schiaffino, S. Holz, W. Belloso y G. Placci. Agradezco a C. Baldovino, A. Horta, P. Tecco. G. Zuquin. V. Aschero, P. Rodríguez, M.D. Juri y a numerosos asistentes durante el trabajo de campo. Ha sido valiosa la información y colaboración de M. Fuguet y G. Montalvo del EJEA y de L. Dalprá e I. Gotz del ESJ. Muchas gracias también al cuerpo de Guardaparques del Parque Nacional Iguazú, especialmente a J. Alonso. W. Maciel y A. Georgopulus. M. Franco y A. Tauro revisaron el manuscrito y realizaron valiosas sugerencias. Este trabajo contó con el apoyo financiero del CONICET (Consejo Nacional de Investigaciones Científicas y Técnicas de Argentina), FVSA (Fundación Vida Silvestre Argentina), WWF - EFN (World Wild Life Fund y Education for Nature) y el Instituto de Ecología de la Universidad Nacional Autónoma de México. Agradezco a los chacreros de la Península de Andresito, especialmente a la familia Werle. Fue invaluable el apoyo financiero y logístico del CIES (Centro de Investigaciones Ecológicas Subtropicales) del Parque Nacional Iguazú. Finalmente, agradezco a los revisores anónimos de la revista por sus valiosos comentarios y sugerencias.

\section{RESUMEN}

Se analizó el efecto del aprovechamiento forestal (maderero y de la extracción de palmitos; Euterpe edulis, Palmae), sobre la estructura, diversidad y composición florística de los bosques denominados palmitales de la Selva Atlántica Argentina ( $54^{\circ} 13^{\prime} \mathrm{W}, 25^{\circ} 41^{\prime} \mathrm{S}$ ), en la provincia de Misiones, Argentina. Se establecieron 3 ha de parcelas permanentes, dos ubicadas en la zona intangible del Parque Nacional Iguazú (PNI) y otra en un sitio aprovechado forestalmente lindante. Se midieron tres parcelas no permanentes cada una de 0.2 ha. Una se ubicó en la zona de reserva del PNI donde hay extracciones ilegales de palmito. Las otras dos estaban en sitios con aprovechamiento forestal. Todos los árboles y palmitos DAP $>10$ $\mathrm{cm}$ fueron identificados y se les midió DAP y altura. Para cada uno de los 6 sitios se estimó riqueza y diversidad de especies arbóreas, composición específica, número 
de especies endémicas y densidad de árboles de especies aprovechables forestalmente. La riqueza y diversidad de especies se correlacionó con la densidad de palmito. Los índices de Shannon de los sitios se compararon con la prueba t y la corrección de Bonferroni. Las estructuras diamétricas considerando todos lo árboles y solo las especies maderables se compararon con la prueba de Duncan. El aprovechamiento de E. edulis aumenta la densidad de otras especies arbóreas, disminuyendo la densidad de palmitos. El aprovechamiento forestal va acompañado de un aumento en la diversidad y en la densidad de especies heliofilas que son las que tienen mayor valor maderero en la región. Este aprovechamiento disminuye la densidad de palmitos, de especies endémicas que normalmente ocurren en bajas densidades y de especies que se encuentran en la lista roja de la UICN. Los resultados sugieren que se puede manejar la estructura del bosque para la producción de maderera y de palmito. La zona intangible del PNI es la que tiene mayor valor para la conservación de la Selva Atlántica ya que en él se encuentran mayor número de endemismos y las especies en peligro de extinción.

Palabras claves: Selva Atlántica, Euterpe edulis, manejo forestal, endemismos, áreas protegidas.

\section{REFERENCIAS}

Begon, M., J.L. Harper \& C.R. Townsend. 1988. Ecología: Individuos, poblaciones y comunidades. Omega, Barcelona, España. 886 p.

Boot, R.G.A., R.E. Gullison. 1995. Approaches to developing sustainable extraction systems for tropical forest products. Ecol Appl. 5: 896-903.

Burley, J. \& I. Gauld. 1995. Measuring and monitoring forest biodiversity: a commentary. Measuring and monitoring biodiversity in Tropical and Temperate Forests, p 19-46. En T.J.B. Boyle \& B. Boontawee (eds). CIFOR-IUFRO. Bogor, Indonesia. 395 p.

Cannon, Ch.H., D.R. Peart \& M. Leighton 1998. Tree species diversity in commercially logged bornean rainforest. Science. 281:1366-1368.

Chao, A. \& T.J. Shen 2003a. Nonparametric estimation of Shannon's index of diversity when there are unseen species in sample. Environ. Ecol. Stat. 10:429-443.

Chediack, S.E. 2006. Servicios ambientales del Parque Nacional Iguazú. Parques Nacionales 3: 14-15.

Crespo, J.A. 1982. Ecología de la comunidad de mamíferos del Parque Nacional Iguazú. Misiones. Rev. M.A.C.N. "Bernardino Rivadavia" 3:1-162, Buenos Aires, Argentina.
Dallmeier, F. 1992. Long-term monitoring of Biological Diversity in tropical forest areas: methods for establishment and inventory of permanent plots. MAB Digest 11. UNESCO, Paris, Francia.26 p.

Devoto, F. \& M. Rothkugel. 1936. Informe sobre los bosques del Parque Nacional Iguazú. Ministerio de Agricultura de la Nación. Sección publicaciones e informes. Buenos Aires, Argentina. 99 p.

Dyer, R.C. 1988. Remote sensing Identification of Tornado Tracks in Argentina. Brazil and Paraguay. Photometric Engineering and Remote sensing 54:1429-1435.

Galindo-Leal, C. \& I. de Gusmao Câmara. 2003. Atlantic Forest hotspot status: An overview, p. 3-11. En C. Galindo-Leal. \& I. de Gusmao Câmara(eds). The Atlantic Forest of South America. Biodiversity status, threats and outlook. State of the hotspots. Washington DC, EEUU. 488 p.

Gentry, A. H \& J. Terborgh. 1990. Composition and Dynamics of the Cosha Cashu "Mature" Floodplain Forest, p. 542-564. En A.Gentry (ed). Four Neotrpical Rainforests. Yale University, New Haven, Connecticut, EEUU. 627 p.

Holz, S. \& G. Placci. 2003. Socioeconomic roots of biodiversity loss in Misiones, p. 207- 226. En C. GalindoLeal. \& I. de Gusmao Câmara(eds). The Atlantic Forest of South America. Biodiversity status, threats and outlook. State of the hotspots. Washington DC, EEUU. 488 p.

Lammertink, M. 2004. A Multiple-Site Comparison of Woodpecker Communities in Bornean Lowland and Hill Forests. Conserv. Biol. 18:746-757.

Myers, N. 1988. Threatened biotas: 'hotspots' in tropical forests. Environmentalists 8:187-208.

Myers, N., R.A.Mittermeier \& C.G. Mittermeier. G.A.B. da Fonseca and J. Kent. 2000. Biodiversity hotspots for conservation priorities. Nature 403:853-858.

Panfil, S.N. \& R.E. Gullison. 1998. Short term impacts of experimental timber harvest intensity on forest structure and composition in the Chimanes Forest, Bolivia. Forest. Ecol. Manag. 102:235-243.

Placci, L.G, S.I. Arditi, P.A. Giorgis \& A.A. Wutrich. 1992. Estructura del palmital e importancia de Euterpe edulis como especie clave en el Parque Nacional Iguazú. Argentina. Yvyrareta 3:93-108.

Placci, L.G. \& P.A. Giorgis. 1993. Estructura y diversidad de la selva del Parque Nacional Iguazú. Argentina. Actas de VII Jornadas técnicas de ecosistemas 
forestales nativos: usos, manejo y conservación, p. 123-138. Eldorado, Misiones, Argentina.

Sáenz,G., B. Finegan \& M. Guariguata. 1999. Crecimiento y mortalidad en juveniles de siete especies arbóreas en un bosque muy húmedo tropical intervenido de Costa Rica. Rev. Biol. Trop. 47:45-57.

Schiaffino, K., J. Herrera, S. Chediack \& J. Alonso. 2001. Relevamiento ambiental del establecimiento "El yaguareté". Caracterización ecológica y evaluación de su condición como unidad de conservación y manejo. Programa Refugios de Vida Silvestre de Fundación Vida Silvestre Argentina. Buenos Aires, Argentina. $50 \mathrm{p}$.

Sist, P. 2000. Reduced impact logging in the tropics: objectives, principles and impacts. Int. For. Rev 2:3-10.

Sokal, R.R. \& F.J. Rohlf. 1995. Biometry: the principles and practice of statistics in biological research. Freeman. Nueva York, EEUU. 887 p.

ter Steege, H., R.G.ABoot, L.C Brouwer, J.C. Caesar, R.C. Ek, D.S. Hammond, P.P. Haripersaud, P.van der Hout, V.G. Jetten, A.J.van Kekem, M.A. Kellman, A. Zab Khan, M. Polak, T.L. Pons, J. Pulles, D.Raaimakers, S.A. Rose, J.J.van der Sanden \& R.J.Zagt. 1996. Ecology and logging in a tropical rainforest in Guyana, with recommendations for forest management. Tropenbos Series 14, The Tropenbos Foundation,Wageningen, Reino de los Países Bajos. 123 p.

Wells, K., E. K.V. Kalko, M.B. Lakim \& M. Pfeiffer. 2007. Effects of rain forest logging on species richness and assemblage composition of small mammals in Southeast Asia. J. Biogeogr. 34:1087-1099.

Whitmore, T.C. 1998. An introduction to tropical rainforests. Universidad de Oxford, Londres, Reino Unido. 296 p.

Yodzis, P. 1989. Introduction to theoretical ecology. Harper \& Row, Nueva York, EEUU. 384 p.

Zar, J. 1996. Biostatistical analysis. Prentice-Hall, Nueva Jersey, EEUU. 662 p.
Zuloaga, F. O. \& O. Morrone (eds.). 1999. Catálogo de las Plantas Vasculares de la Argentina. Missouri Botanical Garden 74: 1-1246 (también disponible en-línea: http://www.darwin.edu.ar/Proyectos/ FloraArgentina/FA.asp)

\section{REFERENCIAS DE INTERNET}

Americas Regional Workshop (Conservation \& Sustainable Management of Trees. Costa Rica) 1998. Aspidosperma polyneuron. En: IUCN 2004. 2004 IUCN Red List of Threatened Species. (Revisado: Abril 12, 2006, http://www.iucnredlist.org).

Americas Regional Workshop (Conservation \& Sustainable Management of Trees. Costa Rica) 1998. Balfourodendron riedelianum. En: IUCN 2004. 2004 IUCN Red List of Threatened Species. (Revisado: Abril 12, 2006, http://www.iucnredlist.org).

Americas Regional Workshop (Conservation \& Sustainable Management of Trees. Costa Rica) 1998. Cedrela fissilis. En: IUCN 2004. 2004 IUCN Red List of Threatened Species. (Revisado: Abril 12, 2006, http:// www.iucnredlist.org).

Carvalho. L.d'A.F. 1998. Solanum granulosum-leprosum. En: IUCN 2004. 2004 IUCN Red List of Threatened Species. (Revisado: Abril 12, 2006, http://www. iucnredlist.org).

Chao. A. \& T.J. Shen 2003b. Program SPADE (Species Prediction and Diversity Estimation). Program and User`s Guide published. (Revisado: Enero, 20, 2007, http://chao.stat.nthu.edu.tw).

Prado. D. 1998. Myrocarpus frondosus. En: IUCN 2004. 2004 IUCN Red List of Threatened Species. (Revisado: Abril 12, 2006, http://www.iucnredlist. org).

World Conservation Monitoring Centre 1998. Albizia edwarllii. En: IUCN 2004. 2004 IUCN Red List of Threatened Species. (Revisado: Abril 12, 2006, http:// www.iucnredlist.org). 


\begin{abstract}
ANEXO 1
Lista de familias y especies de árboles con $\mathrm{DAP}<10 \mathrm{~cm}$ registradas en los palmitales de la Provincia de Misiones. Para cada especie se indica el estado de conservación propuesto en la Lista Roja de la UICN (World Conservation Monitoring Centre 1998). Los individuos con EN, corresponden a la categoría de "en peligro". De los árboles que no pudieron ser identificados en el campo se recolectaron ejemplares que se depositaron en el herbario del CIES (Centro de investigaciones ecológicas subtropicales del PNI) y del IBONE (Instituto de Botánica del Noreste Argentino). También se indica si la especie es o no endémica de Selva Atlántica.
\end{abstract}

Familia

Especie

$\begin{array}{ccc}\text { Lista } & \text { Ejemplar } & \text { Endémica } \\ \text { Roja } & \text { de herbario } & \\ \text { UICN } & \text { número } & \end{array}$

Anonaceae

Apocynaceae

Apocynaceae

Apocynaceae

Araliaceae

Araliaceae

Araliaceae

Arecaceae

Arecaceae

Bignoniaceae

Bignoniaceae

Bombacaceae

Boraginaceae

Boraginaceae

Boraginaceae

Caricaceae

Cecropiaceae

Celastraceae

Celtidaceae

Clusiaceae

Euphorbiaceae

Euphorbiaceae

Euphorbiaceae

Euphorbiaceae

Flacourtiaceae
Rollinia emarginata Schltal.

Aspidosperma australe Muell. Arg.

Aspidosperma polyneurum Muell. Arg.

Tabernaemontana catharinensis A. DC.

Dendropanax cuneatus (DC.) Decne. \& Planch.

Pentapanax warmingianus (Marchal) Harms

Schefflera morototoni (Aubl.) Maguire, Steyerm \&

Frodin

Arecastrum romanzoffianum (Cham.) Becc.

Euterpe edulis Mart.

Jacaranda micrantha Cham.

Tabebuia heptaphylla (Vell.) Toledo

Ceiba insignis (Kunth) P.E. Gibbs \& Semir

Cordia ecalyculata Vell.

Cordia trichotoma (Vell.) Arrab. ex Steud

Patagonula americana L.

Jacaratia spinosa (Aubl) A. DC.

Cecropia pachystachya Tréc.

Maytenus ilicifolia Mart. Ex Reissek

no

no

no

si

$\mathrm{si}$

no

no

si

si

no

no

si

no

no

no

$\mathrm{si}$

si

no

Celtis iguanaea (Jacq.) Sarg.

Rheedia brasiliensis (Mart.) Planch. \& Triana

no

Alchornea glandulosa Poepp.

no

Alchornea triplinervia (Spreng.) Muell. Arg.

Sebastiania brasiliensis Spreng.

Tetrorchidium rubrivenium Poepp.\& Endl.

Ejemplar de herbario $\mathrm{H} 243$ no

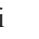

o

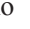

no 
Flacourtiaceae

Flacourtiaceae

Flacourtiaceae

Icacinaceae

Lauraceae

Lauraceae

Lauraceae

Lauraceae

Lauraceae

Lauraceae

Ocotea sp. 3

Leguminosae-Cae.

Apuleia leiocarpa (Vogel) J.F. Macbr.

Leguminosae-Cae.

Holocalyx balansae M. Micheli

Leguminosae-Cae.

Peltophorum dubium (Sprengel) Taub.

Leguminosae-Mim. Acacia tucumanensis Griseb.

Leguminosae-Mim. Albizia edwallii (Hoehne) Barneby \& Grimes

Leguminosae-Mim. Albizia niopoides (Spuce ex Benth.) Burk.

Leguminosae-Mim. Enterolobium contortisiliquum (Vell. Conc.) Morong.

Leguminosae-Mim. Inga semialata (Vell.) Mart.

Leguminosae-Mim. Inga uruguensis Hook. et Arn.

Leguminosae-Mim. Parapiptadenia rigida (Benth.) Brenan

Leguminosae-Pap. Erythrina falcata Benth.

Leguminosae-Pap. Lonchocarpus leucanthus Burk.

Leguminosae-Pap. Lonchocarpus muehlbergianus Hassl.

Leguminosae-Pap. Machaerium minutiflorum Tul.

Leguminosae-Pap. Machaerium paraguariense Hassl.

Leguminosae-Pap. Myrocarpus frondosus Allemao

Malvaceae

Meliaceae

Meliaceae

Meliaceae

Meliaceae

Meliaceae
Bastardiopsis densiflora (Hook. \& Arn.) Hassl.

Cabralea canjerana (Vell.) Mart.

Cedrela fissilis Vell.

Guarea kunthiana A. Juss.

Trichilia catigua A. Juss.

Trichilia claussenii D.C. no

Ejemplar de herbario $\mathrm{H} 322$

\section{no}

no

no

no

no

no

no

Arboles número 59 y 1012 de las parcelas permanentes

Ejemplar de herbario H324 
Moraceae

Ficus sp.

Moraceae

Moraceae

Myrsinaceae

Myrsinaceae

Myrtaceae

Myrtaceae

Myrtaceae

Myrtaceae

NN

Nyctaginaceae

Nyctaginaceae

Proteaceae

Rosaceae

Rubiaceae

Rutaceae

Rutaceae

Rutaceae

Rutaceae

Rutaceae

Sapindaceae

Sapindaceae

Sapindaceae

Sapotaceae

Sapotaceae

Simaroubaceae

Solanaceae

Solanaceae

Solanaceae

Solanaceae

Solanaceae

Styracaceae Boer

NN 1

Radlk.
Maclura tinctoria (L.) Steud.

Posiblemente varias especies

Sorocea bonplandii (Baill.) W.C.Burger, Lanj. \& Wess.

Myrsine laetevirens (Mez.) Arechav.

Myrsine umbellata Mart.

Britoa guazumifolia

Campomanesia xanthocarpa $\mathrm{O}$. Berg.

Eugenia pyriflora

Plinia rivularis (Cambess.) Rotman

Pisonia aculeata L.

Pisonia zapallo Griseb.

Roupala brasiliensis Klotzsch

Prunus brasiliensis (Cham.\&Schltdl.) D. Dietr.

Fagara hyemalis (A. St.-Hil.) Engl.

Fagara riedeliana (Engl.) Engl.

no

si

Helietta apiculata Benth.

Pilocarpus pennatifolius Lem.

Allophylus edulis (A. St. Hil., A. Juss \& Cambess.)

Cupania vernalis Cambess.

Diatenopteryx sorbifolia Radlk.

Chrysophyllum gonocarpum (Mart.\& Eichler) Engl.

Chrysophyllum marginatum (Hook.\& Arn.) Radlk.

Ejemplar de herbario $\mathrm{H} 264$

Cestrum euanthes Schltdl.

Cestrum $s p$

Solanum granulosum-leprosum Dunal

$\mathrm{LR} / \mathrm{cd}$

Solanum sanctae-catarinae Dunal

Solanum sp.

Ejemplar de herbario $\mathrm{H} 241$ 


\section{Tiliaceae}

Tiliaceae

Urticaceae

Verbenaceae

Verbenaceae

Varias especies

NN 2
Luehea candicans Mart.

Luehea divaricata Mart.

Urera baccifera (L:) Gaudich.

Aegiphila hassleri Briq.

Vitex megapotamica (Spreng.) Moldenke

Lianas

NN EJEA no

no

no

si

no 